the leg, and the 'wounds dressed with tr. benz. co. On May 18th, the wounds being healed, each foot was brought again to a right angle with the leg with Thomas's wrench. Each foot and leg was massaged daily and Scarpa's shoe applied for twelve days. Then boots and steels were applied to the feet and legs (one steel on the inner side of the leg was applied in this case) as shown in Fig. 3.

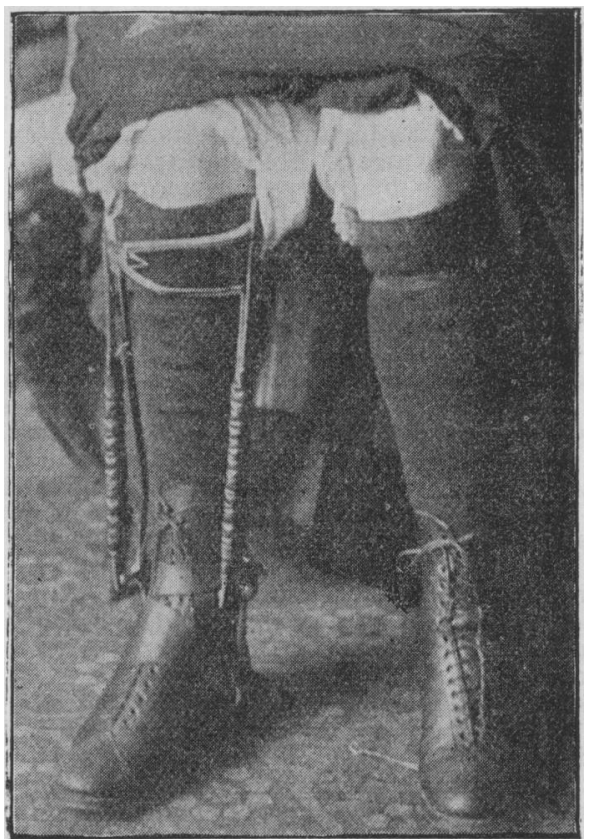

Fig. 3, Case 1.-Talipes equinus, right. Operated on May 1st, 1891. Steel and boot applied June 5th, 1891.

By wearing the boots and steels the patient, in a few days, could walk well without help. He left for home to-day, with the instruction to massage his legs daily. He has walked daily with ease and comfort since then. ${ }^{3}$

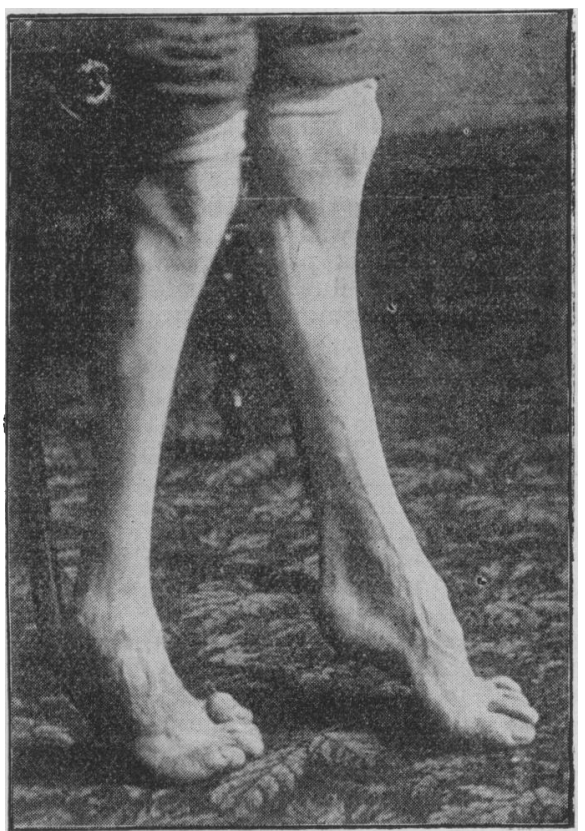

Fig. 4, Case 3.-H. R., aged 27 jears. Double talipes equino-varus; ailing 20 years.

This case, and casts of the limb of Cases I and II, taken before und four weeks after operation, were shown at the meeting.
The operations in the cases here described were conducted on the simplest principles. (1) The limbs were rendered bloodless by applying Esmarch's bandage. (2) Methylene was used as an anæsthetic in two cases, and in the third case ether spray was applied locally. I consider methylene is the best anæsthetic in these short operations. (3) The contracted plantar fascia and tendons on the sole of the foot were cut subcutaneously, one week before the tendo Achillis, because the toes and anterior part of the foot can be more thoroughly straightened by the "wrench," while the heel is held firmly by the tendo Achillis. (4) Each foot was thoroughly massaged and forcibly straightened and put in proper position daily (after the skin wound had healed) for three weeks, and in one of the patients for four weeks after the operation. Thomas's wrench I found a most useful instrument for forcibly stretching the foot after operation. (5) The patients were recommended to walk as much as possible every day, and continue the massage for at least two years after operation.

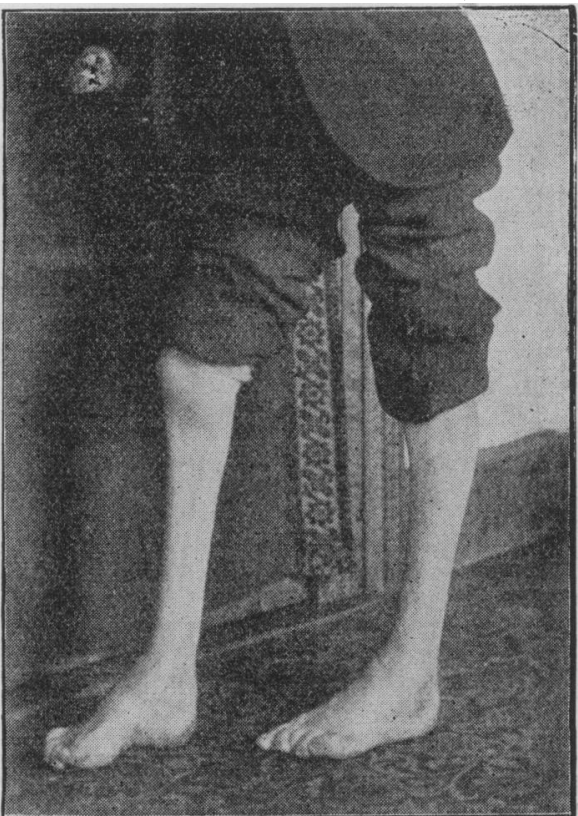

Fjg. 5, Case 3.-H. R., aged 27 years. Double talipes equino-varus, four weeks after operation.

\section{CANCER OF THE BREAST.}

BY CHARLES E. JENNINGS, M.D., M.S., F.R.C.S.

THE case which forms the basis of this communication is that of an unmarried patient (aged 57), under the care of Dr. J. R. Philpots. The patient had complained of severe pain in her left breast during the autumn of 1892 ; and upon examination, on November 19th, both breasts were found of an unusually large size, with a certain amount of irregular induration. In parts of either breast there was toughness, as though small pieces of whipcord had been inserted; but these signs were more marked in the left than in the right breast, and the left nipple was not so prominent as the right one. Dr. Philpots proposed that a portion of the left breast should be excised and submitted to microscopic examination. Chloroform having been administered, two portions of indurated mammary tissue, the size of nuts, were removed from the central and deeper part of the left organ.

Microscopic sections of the specimens have since been furnished to me by Mr. Stanley Boyd and Mr. E. H. Fricke, of Charing Cross Hospital. I am also indebted to Mr. Francis Fowke for his kindness in procuring an excellent photograph

4 My sincere thanks are due to Mr. Adam Sloan, instrument maker, Belfast, for the careful manner in which he made the boots and steels for these patients. 
of one of the specimens. In some of the sections, glandular acini and ducts of nearly normal appearance can be seen, the acini being lined with a layer of low cylindrical cells, but in parts, as illustrated, the contour of the acini is broken and lost, and the acini are hyperdistended with cells of irregular shape from mutual pressure which have infiltrated the surrounding stroma. The stroma is unusually dense in most parts of the sections. These changes are commonly accepted as conclusive of scirrhous cancer, and demonstrate the peculiarly insidious manner in which the disease arises from small foci concealed from detection by manipulative examination by superficial coverings of apparently healthy mammary tissue.

I should like to call attention to certain bodies of which I do not know the import in these sections. Scattered throughout the stroma are bodies shaped like bacilli, but much too large for them. They are longer than the diameter of a cancer cell and their width is about a quarter or a fifth of their length.

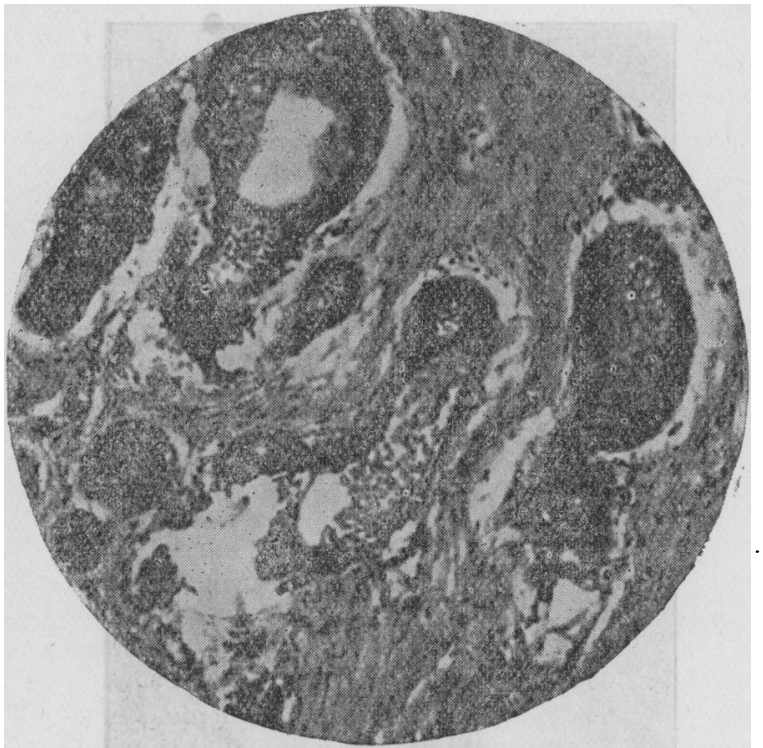

In such a case as this the value of microscopic examination cannot be overestimated, as affording a certain diagnosis at a much earlier period of the disease than can be arrived at by other methods. Excepting those rare cases in which mammary cancer undergoes spontaneous resolution, it will scarcely be disputed that the prognosis, whether favourable or the reverse, bears a direct ratio to the period in the natural course of the disease in which it is detected and submitted to surgical treatment. If detected in the precancerous stage or when cancer has only first made a definite beginning, as in this example, removal of the affected organ will rarely be followed by recurrence, in proof whereof $I$ could cite several instances; but if the disease be allowed to proceed unchecked till it assumes considerable proportions, how can it be hoped that some outlying portion of cancerous deposit of minute size will not be left behind even after an operation of great magnitude?

\section{PANCREATIC CYST OR. EFFUSION INTO LESSER PERITONEAL CAVITY?}

By PAUL SWAIN, F.R.C.S.,

Surgeon to the South Devon and East Cornwall Hospital, Plymouth.

As a sequel to the cases of effusion into the lesser peritoneal cavity recorded by Mr. Jordan Lloyd in the BrITISH MrdicaI JounNaL for November 12th, 1892, the following case is of much interest:

History.-C. N., a man aged 38, came under my care on September 22nd, 1892, with the following history: Three months previously he had an attack of colic with pain in the right side of the abdomen. The bowels were not opened for three days, and at this time a lump was found by a naval surgeon in the abdomen. He got over this attack and resumed work, but four or five weeks ago, while running a race, he strained himself and was seized soon after with great pain in the abdomen. For this he was admitted to the naval hospital, and after seven weeks invalided out of the service.

Present State.-It was at this time that I first saw him, and found him in the following condition: A fine, healthylooking man, with a swelling in the abdomen, which gave rise to no pain beyond a feeling of uneasiness after exercise and some fulness after meals. He had lost 2 stones in weight in three months. He had never been out of England, and always enjoyed particularly good health. Temperature $98^{\circ} \mathrm{F}$.; pulse 60 ; respiration 18 . The abdomen was distended between the umbilicus and xiphoid cartilage by a round, elastic, fluctuating, and tense smooth swelling, at once felt and easily defined by placing the hands on the abdomen. It appeared to be about the size of an ordinary cocoa-nut, and had well-marked pulsation, which seemed to be expansile, and yet on pressing the hands deeply in on each side and lifting the cyst forward the pulsation became less marked. The percussion note over the swelling was dull, the dull area being almost exactly central, but inclining slightly to the left. The heart was normal in position, and the sounds were clear. The femoral and tibial pulses were good and equal on the two sides. On auscultating the swelling a bruit was to be heard over a very limited area in the centre. All the other organs were healthy. Digestion was good, the tongue clean; there was nothing unusual in the appearance of the motions; had occasional sickness lately.

Diagnosis. - The diagnosis seemed to be between (1) pancreatic cyst, (2) aneurysm of some branch of the coliac axis, (3) cystic disease of mesenteric glands ; and considering the absence of all symptoms except occasional colic and sickness, and the sound state of all other organs, the former seemed the most likely, so I decided to open the abdomen, and deal with it as circumstances directed.

Operation.-On October 8th, chloroform and ether having been administered, I opened the peritoneal cavity by a central incision 3 inches long, midway between the umbilicus and ensiform cartilage. The omentum at once bulged into the wound and was turned up, but, as the tumour could not be reached well from below, it was reduced again; the stomach was drawn down and to the left, and the tumour bulged forward above it, being bounded above by the liver, below by the stomach, and passing deeply back. Now on grasping the mass it was evident there was not expansile pulsation, so sponges were packed round it, and a small trocar thrust in. A stream of dirty brown fluid spurted out to a distance of several feet; the trocar was then withdrawn and the opening enlarged, when the cavity was found to extend back to the vertebral column, where its posterior wall was firmly fixed. After removal of all the fluid a considerable quantity of greenish-black masses of pigment was found lining the interior, and as much of this as possible removed by means of the finger nail and flushing. A Keith's glass drainage tube was then introduced, the lower part of the wound closed, and the edges of the opening in the cyst sutured to the upper angle of the abdominal wound. The end of the glass drain was then passed throught a slit in a sheet of thin rubber, and the remainder of the wound dressed underneath. A sponge, wrung out of an antiseptic solution, was placed over the mouth of the drain. Owing to the fixity of the cyst wall posteriorly, and its brittleness, some difficalty was found in bringing it forward enough to suture to the skin edges.

After-history.-Recovery was uninterrupted, and the only trouble lay in the tendency of the cavity to close in the middle, leaving a pouch posteriorly in which fluid collected. In twenty-four hours the cyst, which contained 30 ounces, had contracted so that it gripped the tube tightly. The sinus completely healed in less than two months. On September 30 th, before operation, his weight was 9 st. $103 \mathrm{lbs}$.; November 11th, 10 st. 4 lbs.; December 7th, -11 st. -3 lbs.

Examination of Fluid and Sac Wall.-The fluid measured 30 ounces, was alkaline, of a dirty-hrown colour, the solid matter in it consisting of blood cells, a few large (?) colostrum cells, and 